\title{
Evolução Clínica de Pacientes Diabéticos Tratados por Intervenção Coronária Percutânea Utilizando Stents Com e Sem Eluição de Fármacos
}

\author{
Edgar Stroppa Lamas ${ }^{1}$, Antonio de Castro Filho², Marinella Patrizia Centemero ${ }^{3}$, Henrique Chigueo Iwace ${ }^{4}$, \\ Fausto Feres ${ }^{5}$, Rodolfo Staico ${ }^{6}$, Dimytri Siqueira ${ }^{7}$, J. Ribamar Costa Jr. ${ }^{8}$, Ricardo Costa ${ }^{9}$, \\ Daniel Chamié ${ }^{10}$, Áurea J. Chaves ${ }^{11}$, Amanda G. M. R. Sousa ${ }^{12}$, Alexandre Abizaid ${ }^{13}$
}

\begin{abstract}
RESUMO
Introdução: A revascularização percutânea de diabéticos é frequente e a utilização de stents farmacológicos (SF) é desejável, pois estes reduzem a reestenose e a necessidade de nova revascularização. O objetivo desse estudo foi comparar os resultados clínicos de longo prazo entre diabéticos tratados com e sem SF. Métodos: Analisou-se uma coorte consecutiva de diabéticos submetidos à intervenção coronária percutânea (ICP) entre janeiro de 2009 e dezembro de 2012, em hospital terciário da rede pública. Esses pacientes foram acompanhados prospectivamente. Resultados: Avaliamos 939 diabéticos, sendo $359(38,3 \%)$ tratados com SF e $580(61,7 \%)$ tratados com stents não farmacológicos (SNF). A taxa de eventos cardiovasculares adversos maiores (ECAM) em 12,6 \pm 3,4 meses foi maior no grupo SNF $(9,5 \%$ vs. $14,8 \%$; risco relativo $R R=1,56$; intervalo de confiança de $95 \%$ - IC 95\% 1,07-2,27; $\mathrm{P}=0,02)$, assim como o óbito $(2,8 \%$ vs. $6,7 \% ; \mathrm{RR}=2,41 ; \mathrm{IC}$ $95 \% 1,22-4,77 ; \mathrm{P}<0,01)$ e a revascularização do vaso alvo $(3,9 \%$ vs. $7,2 \% ; R R=1,85 ; \mathrm{IC} 95 \% 1,03-3,35 ; \mathrm{P}=0,04)$. Não foram observadas diferenças na incidência de infarto do miocárdio (1,7\% vs. 0,5\%; RR =0,30; IC 95\% 0,07-1,23; $P=0,08)$ ou acidente vascular encefálico $(1,1 \%$ vs. $0,2 \%$; $R R=0,15 ; I C$ 95\% 0,01-1,37; $P=0,07)$. A análise multivariada revelou que a doença renal crônica $(R R=2,05$; IC 95\% $1,40-2,98 ; \mathrm{P}<0,01)$ e a síndrome coronária aguda $(\mathrm{RR}=2,08$; IC 95\% 1,42-3,02; $\mathrm{P}<0,01)$ foram os únicos preditores independentes de ECAM. Conclusões: Em pacientes diabéticos não selecionados, a evolução clínica tardia foi pior para os
\end{abstract}

\section{ABSTRACT}

\section{Clinical Outcome of Diabetic Patients Treated by Percutaneous Coronary Intervention Using Drug-Eluting and Bare Metal Stents}

Background: Percutaneous revascularization in diabetic is frequent and the use of drug-eluting stents (DES) is desirable, reducing restenosis and the need for repeat revascularization. The objective of this study was to compare the long-term outcomes of diabetic patients treated with and without DES. Methods: A consecutive cohort of diabetic patients undergoing percutaneous coronary intervention ( $\mathrm{PCI}$ ) between 01/2009 and $12 / 2012$ in a public tertiary hospital was prospectively followed-up. Results: Nine hundred and thirty-nine diabetic patients $(38.3 \%)$ treated with DES and $580(61.7 \%)$ treated with bare metal stents (BMS) were evaluated. The rate of major adverse cardiac events (MACE) in $12.6 \pm 3.4$ months was greater in the BMS group $(9.5 \%$ vs. $14.8 \% ; \mathrm{RR}, 1.56 ; 95 \%$ $\mathrm{Cl}, 1.07-2.27 ; \mathrm{P}=0.02)$, as well as death $(2.8 \%$ vs. $6.7 \%$; $\mathrm{RR}, 2.41 ; 95 \% \mathrm{Cl}, 1.22-4.77 ; \mathrm{P}<0.01)$, and target vessel revascularization $(3.9 \%$ vs. $7.2 \%$; RR, $1.85 ; 95 \% \mathrm{Cl}, 1.03-3.35$; $\mathrm{P}=0.04)$. There were no differences in the incidence of myocardial infarction $(1.7 \%$ vs. $0.5 \%$; RR, $0.30 ; 95 \% \mathrm{Cl}$, $0.07-1.23 ; \mathrm{P}=0.08)$ or stroke $(1.1 \%$ vs. $0.2 \%$; RR, 0.15 ; $95 \% \mathrm{Cl}, 0.01-1.37 ; \mathrm{P}=0.07)$. Multivariate analysis indicated that chronic kidney disease (RR, 2.05; $95 \% \mathrm{Cl}, 1.40-2.98$; $\mathrm{P}<0.01)$ and acute coronary syndrome $(\mathrm{RR}, 2.08 ; 95 \% \mathrm{Cl}$ $1.42-3.02 ; \mathrm{P}<0.01)$ were the only independent predictors

\footnotetext{
${ }^{1}$ Médico residente do Serviço de Cardiologia Invasiva do Instituto Dante Pazzanese de Cardiologia. São Paulo, SP, Brasil.

${ }^{2}$ Médico residente do Serviço de Cardiologia Invasiva do Instituto Dante Pazzanese de Cardiologia. São Paulo, SP, Brasil.

${ }^{3}$ Doutora. Cardiologista do Serviço de Cardiologia Invasiva do Instituto Dante Pazzanese de Cardiologia. São Paulo, SP, Brasil.

${ }^{4}$ Médico residente de cardiologia do Instituto Dante Pazzanese de Cardiologia. São Paulo, SP, Brasil.

${ }^{5}$ Doutor. Cardiologista intervencionista do Serviço de Cardiologia Invasiva do Instituto Dante Pazzanese de Cardiologia. São Paulo, SP, Brasil. ${ }^{6}$ Doutor. Cardiologista intervencionista do Serviço de Cardiologia Invasiva do Instituto Dante Pazzanese de Cardiologia. São Paulo, SP, Brasil. ${ }^{7}$ Doutor. Cardiologista intervencionista do Serviço de Cardiologia Invasiva do Instituto Dante Pazzanese de Cardiologia. São Paulo, SP, Brasil. ${ }^{8}$ Doutor. Cardiologista intervencionista do Serviço de Cardiologia Invasiva do Instituto Dante Pazzanese de Cardiologia. São Paulo, SP, Brasil.
}

\footnotetext{
${ }^{9}$ Doutor. Cardiologista intervencionista do Serviço de Cardiologia Invasiva do Instituto Dante Pazzanese de Cardiologia. São Paulo, SP, Brasil. ${ }^{10}$ Cardiologista intervencionista do Serviço de Cardiologia Invasiva do Instituto Dante Pazzanese de Cardiologia. São Paulo, SP, Brasil.

${ }^{11}$ Doutora. Cardiologista do Serviço de Cardiologia Invasiva do Instituto Dante Pazzanese de Cardiologia. São Paulo, SP, Brasil.

${ }^{12}$ Livre-docente. Diretora Geral do Instituto Dante Pazzanese de Cardiologia. São Paulo, SP, Brasil.

${ }^{13}$ Livre-docente. Diretor da Divisão de Cardiologia Invasiva do Instituto Dante Pazzanese de Cardiologia. São Paulo, SP, Brasil.
}

Correspondência: Marinella Patrizia Centemero. Serviço de Cardiologia Invasiva do Instituto Dante Pazzanese de Cardiologia. Avenida Dante Pazzanese, 500 - Vila Mariana - São Paulo, SP, Brasil - CEP 04012-180 E-mail: mpcentemero@yahoo.com.br

Recebido em: 19/9/2013 • Aceito em: 4/12/2013 
tratados com SNF. Após o ajuste das variáveis de confusão, o uso de SF não se mostrou preditor independente da redução de ECAM.

Descritores: Intervenção coronária percutânea. Diabetes mellitus. Stents farmacológicos. Doença da artéria coronariana.

A doença arterial coronária (DAC) é responsável por, aproximadamente, $75 \%$ das mortes cardiovasculares em diabéticos. ${ }^{1}$ Uma parte considerável desses pacientes é submetida à intervenção coronária percutânea (ICP), seja na vigência de quadros agudos ou para o tratamento da doença estável, sendo que os diabéticos correspondem, atualmente, a aproximadamente 30\% daqueles revascularizados percutaneamente. ${ }^{2}$

Nesses pacientes, a DAC possui características peculiares, seja pela associação de comorbidades, que elevam o risco clínico, seja pela presença de doença aterosclerótica mais extensa e complexa, com vasos de menor calibre e maior propensão à trombose. Especificamente em relação à $\mathrm{ICP}$, esse subgrupo apresenta maior tendência à reestenose coronária pós-implante de stents e também maior ocorrência de outros eventos cardíacos adversos na evolução tardia, como morte e infarto agudo do miocárdio (IAM). ${ }^{3-5}$

Por sua vez, os stents farmacológicos (SF) já comprovaram sua eficácia na redução das taxas de reestenose e da necessidade de procedimentos adicionais de revascularização, quando comparados aos stents não farmacológicos (SNF), tanto na população global como no subgrupo de diabéticos. ${ }^{6,7}$ Nestes, as próteses com eluição de fármacos também já foram amplamente investigadas, demonstrando altas taxas de sucesso, baixo índice de complicações agudas, além de redução expressiva de novas revascularizações, comparativamente aos SNF. ${ }^{8}$

Entretanto, os resultados relacionados aos desfechos cardiovasculares maiores de segurança, como óbito, IAM e acidente vascular encefálico (AVE), em médio e longo prazos, ainda são contraditórios. ${ }^{7-9}$ Alguns registros internacionais revelam redução de mortalidade e IAM a favor dos SF em diabéticos, tanto na fase inicial como tardia, ${ }^{10}$ benefícios estes não observados em outras séries. ${ }^{11,12}$ Estudos randomizados mais recentes, que compararam essas próteses com a cirurgia de revascularização miocárdica em diabéticos multiarteriais, também não evidenciaram resultados favoráveis aos SF. ${ }^{13,14}$

Em nosso meio, no qual a maioria dos pacientes portadores de DAC é tratada na rede pública de saúde, submetida ao contingenciamento de recursos financeiros dispensados ao Sistema Único de Saúde (SUS), a aquisição de SF para uso universal é inexequível. Nesse contexto, a disponibilização desses dispositivos, com of MACE. Conclusions: In non-selected diabetic patients the long-term clinical outcome was worse for patients treated with BMS. After adjusting for confounding variables, the use of DES was not an independent predictor of reduced MACE.

DESCRIPTORS: Percutaneous coronary intervention. Diabetes mellitus. Drug-eluting stents. Coronary artery disease.

custo muito superior àquele dos SNF, poderia ocorrer para o tratamento de subgrupos portadores de anatomia coronária mais complexa, como os diabéticos.

Em virtude dessas considerações, tivemos por objetivo investigar a evolução clínica tardia de pacientes diabéticos tratados por ICP, com e sem implante de SF.

\section{MÉTODOS}

Foi analisada uma coorte consecutiva composta por diabéticos submetidos à ICP, no período de janeiro de 2009 a dezembro de 2011, no Instituto Dante Pazzanese de Cardiologia, em São Paulo (SP), um hospital terciário da rede pública, com ampla experiência em procedimentos percutâneos.

O objetivo primário foi comparar as taxas de eventos cardiovasculares adversos maiores (ECAM), ou seja, óbito, AVE, IAM e revascularização do vaso alvo (RVA) entre os diabéticos tratados com e sem SF. O objetivo secundário foi avaliar as taxas de ocorrência de cada evento clínico isolado nos dois grupos, assim como identificar possíveis preditores independentes de ECAM.

\section{Definições}

Diabetes mellitus foi diagnosticado segundo os critérios da American Diabetes Association, que estabelece a presença de valores de glicemia de jejum $\geq 126 \mathrm{mg} / \mathrm{dL}$, ou hemoglobina glicada $\geq 6,5 \%$, ou glicemia $\geq 200 \mathrm{mg}$, 2 horas após sobrecarga de glicose. O óbito foi definido como qualquer morte, independentemente da causa, e o óbito cardíaco como morte secundária a causas cardíacas imediatas (IAM, insuficiência cardíaca e arritmia fatal), além daquelas mortes não testemunhadas e/ou de causas desconhecidas.

O diagnóstico de IAM periprocedimento foi estabelecido quando se observou elevação da CKMB massa $>3$ vezes o limite superior do valor normal, associada a sintomas indicativos de isquemia miocárdica ou à presença de novas ondas $Q$ no eletrocardiograma pós-procedimento, ou a alterações da contratilidade regional em exames de imagem durante a internação hospitalar. Já o IAM após a alta hospitalar foi considerado quando ocorreu elevação e queda dos níveis de troponina ou CKMB massa, associadas a sintomas típicos de isquemia ou alterações eletrocardiográficas (novas ondas Q/supra ou infradesnivelamentos do segmento ST), ou necessidade de revascularização de 
urgência, ou achados anatomopatológicos compatíveis com isquemia aguda.

O AVE foi definido como a ocorrência de infarto cerebral (AVE isquêmico) ou hemorragia intracerebral e subaracnoidea (AVE hemorrágico), com sintomas persistindo $>24$ horas ou que resultasse em morte $<24$ horas.

A RVA foi considerada como a realização de novo procedimento percutâneo ou cirúrgico após a alta hospitalar para tratar o vaso alvo, na presença de angina ou equivalente clínico sugestivo de isquemia, ou provas funcionais com resultados alterados.

A trombose do stent foi definida de acordo com os critérios do Academic Research Consortium (ARC) como definitiva, se confirmada por achados angiográficos (fluxo TIMI 0 com oclusão do stent por trombo/fluxo TIMI 1, 2 ou 3 e presença de trombo) ou anatomopatológicos; ou provável, definida como qualquer morte inexplicada dentro dos primeiros 30 dias após ICP ou qualquer IAM relacionado ao vaso tratado sem confirmação angiográfica e na ausência de outras causas. ${ }^{15}$

\section{Procedimento}

O procedimento percutâneo foi realizado segundo a prática habitual, recomendada pelas diretrizes nacionais e internacionais. ${ }^{16,17}$ A escolha da via de acesso (femoral ou radial) ficou a cargo do operador, assim como a utilização de inibidores da glicoproteína IIb/IIla.

A dupla antiagregação plaquetária consistiu em aspirina, na dose de $100 \mathrm{mg} /$ dia naqueles em uso crônico, e 300 mg (dose de ataque) nos não previamente tratados. O clopidogrel foi o inibidor da P2Y12 mais frequentemente utilizado, nas doses de ataque de 300 a $600 \mathrm{mg}$ pré-procedimento e, posteriormente, $75 \mathrm{mg} / \mathrm{dia}$ associado à aspirina $100 \mathrm{mg} /$ dia por 1 ano.

A decisão de utilizar SF ficou a critério do operador e condicionada à disponibilidade dos mesmos na época do estudo, considerando os seguintes cenários: vasos $\leq 2,5 \mathrm{~mm}$, lesões > $20 \mathrm{~mm}$ e reestenose intra-stent; expectativa de não aderência à dupla antiagregação plaquetária em 1 ano; presença de diáteses hemorrágicas, doenças hematológicas ou história prévia e recente $(<6 \mathrm{~m})$ de sangramentos digestivos, urológicos ou outros, que pudessem inviabilizar a utilização da dupla antiagregação plaquetária pelo tempo preconizado.

\section{Seguimento clínico}

O acompanhamento clínico foi prospectivo, realizado por meio de consultas médicas, com revisão dos prontuários, uma vez que esse hospital terciário possui um ambulatório próprio para acompanhamento de pacientes submetidos à ICP. Também foram utilizados contatos por telefone, e-mail, telegrama ou relato dos médicos assistentes que encaminharam os pacientes ao nosso serviço.

\section{Análise estatística}

As variáveis contínuas foram analisadas como médias \pm desvio padrão e comparadas usando o teste de Mann-Whitney. As variáveis categóricas foram comparadas usando-se o teste qui-quadrado ou exato de Fisher, quando apropriado.

Análise de sobrevivência livre de eventos foi obtida utilizando as curvas de Kaplan-Meier e comparações foram realizadas pelo método de log-rank.

Modelos de regressão multivariada de Cox foram derivados para ECAM, e as variáveis consideradas para inclusão nos modelos multivariados foram idade, sexo, uso de insulina, insuficiência renal crônica (IRC), síndrome coronária aguda (SCA), ICP prévia, número de vasos tratados, uso de SF e IAM periprocedimento. O nível de significância para permanecer no modelo foi ajustado para 0,01.

Para realização das análises, foi utilizado o programa Statistical Package for the Social Sciences (SPSS) 20.0 e o valor de $\mathrm{P}<0,05$ foi considerado significante.

\section{RESULTADOS}

Foram analisados 939 diabéticos consecutivos, sendo 359 pacientes (38,3\%) tratados com SF e 580 pacientes $(61,7 \%)$ com SNF. A média de seguimento nos dois grupos foi de 12,9 $\pm 3,6$ meses ( 1 a 13 meses) e 12,4 $\pm 2,5$ meses ( 1 a 14 meses), respectivamente, sendo que $80 \%$ dos pacientes tiveram acompanhamento mínimo de 1 ano.

As características demográficas e clínicas desses pacientes revelaram maior proporção de pacientes com fatores de risco para aterosclerose, IAM prévio, insuficiência cardíaca, ICP prévia, e quadros clínicos estáveis nos diabéticos tratados com SF, ao passo que pacientes com doença renal crônica e SCA foram mais frequentes naqueles tratados com SNF. Em relação ao tratamento farmacológico pré-procedimento, os pacientes tratados com SF faziam uso mais frequentemente de betabloqueadores, estatinas e insulina (Tabela 1).

No grupo SF, a artéria descendente anterior foi o vaso mais frequentemente tratado $(50,7 \%)$, ICP de múltiplos vasos foi mais frequente $(25,3 \%$ vs. $5,7 \%$; $\mathrm{P}<0,01)$, redundando numa relação vaso tratado por paciente/stent por paciente também significativamente maior nesse grupo. Também observamos porcentual significativamente maior de lesões em bifurcações e lesões reestenóticas tratadas com SF (Tabela 2). Foram utilizados stents com eluição de zotarolimus (65\%), sirolimus $(31 \%)$, paclitaxel $(2,5 \%)$ e novolimus $(1,5 \%)$.

Em relação aos resultados hospitalares, a taxa de sucesso clínico da ICP foi semelhante nos dois grupos $(97,2 \%$ vs. $95,6 \% ; P=0,22)$, não havendo diferenças na ocorrência de óbito ( 0 vs. $0,7 \%$; $P=0,30$ ) e de AVE $(0$ vs. $0,2, P=0,99)$. O grupo dos pacientes tratados 
TABELA 1

Características clínicas e demográficas dos pacientes

\begin{tabular}{|c|c|c|c|}
\hline Características & $\begin{array}{c}\text { SF } \\
(n=359)\end{array}$ & $\begin{array}{c}\text { SNF } \\
(n=580)\end{array}$ & $\begin{array}{l}\text { Valor } \\
\text { de P }\end{array}$ \\
\hline Idade, anos & $63,0 \pm 10,6$ & $63,2 \pm 10,5$ & 0,80 \\
\hline Sexo feminino, $\mathrm{n}(\%)$ & $145(40,4)$ & $219(37,8)$ & 0,42 \\
\hline Hipertensão arterial, n (\%) & $337(93,9)$ & $533(91,9)$ & 0,30 \\
\hline Dislipidemia, n (\%) & $314(87,5)$ & $453(78,1)$ & $<0,01$ \\
\hline Tabagismo, n (\%) & $204(56,8)$ & $62(10,7)$ & $<0,01$ \\
\hline ICP prévia, n (\%) & $104(29,0)$ & $64(11,0)$ & $<0,01$ \\
\hline CRM prévia, n (\%) & $35(9,7)$ & $88(11,0)$ & 0,17 \\
\hline IAM prévio, n (\%) & $163(45,4)$ & $224(38,6)$ & 0,04 \\
\hline AVE ou AIT prévio, n (\%) & $13(3,6)$ & $18(3,1)$ & 0,70 \\
\hline História familiar de DAC, n (\%) & $73(20,3)$ & $52(9,0)$ & $<0,01$ \\
\hline DPOC, n (\%) & $4(1,1)$ & $10(1,7)$ & 0,58 \\
\hline IRC não dialítica, $\mathrm{n}(\%)$ & $27(7,5)$ & $188(32,4)$ & $<0,01$ \\
\hline DAOP, n (\%) & $16(4,5)$ & $16(2,8)$ & 0,19 \\
\hline Insuficiência cardíaca, n (\%) & $127(35,4)$ & $9(1,6)$ & $<0,01$ \\
\hline Quadro clínico, n (\%) & & & $<0,01$ \\
\hline Síndrome coronária aguda & $36(10,0)$ & $174(30,0)$ & \\
\hline Angina estável & $218(60,7)$ & $268(46,2)$ & \\
\hline Assintomático & $105(29,2)$ & $138(23,8)$ & \\
\hline \multicolumn{4}{|l|}{ Tratamento farmacológico, n (\%) } \\
\hline Insulina & $106(29,5)$ & $58(10,0)$ & $<0,01$ \\
\hline Hipoglicemiante oral & $313(87,2)$ & $520(89,7)$ & 0,24 \\
\hline Ácido acetilsalicílico & $357(99,4)$ & $568(97,9)$ & 0,09 \\
\hline Estatinas & $357(99,4)$ & $537(92,4)$ & $<0,01$ \\
\hline IECA/BRA & $336(93,6)$ & $536(92,4)$ & 0,51 \\
\hline Betabloqueadores & $343(95,5)$ & $477(80,5)$ & $<0,01$ \\
\hline
\end{tabular}

SF: stents farmacológicos; SNF: stents não farmacológicos; ICP: intervenção coronária percutânea; CRM: cirurgia de revascularização miocárdica; IAM: infarto agudo do miocárdio; AVE: acidente vascular encefálico; AIT: ataque isquêmico transitório; DAC: doença arterial coronária; DPOC: doença pulmonar obstrutiva crônica; IRC: insuficiência renal crônica; DAOP: doença arterial obstrutiva periférica; IECA/BRA: inibidores da enzima de conversão/bloqueadores dos receptores de angiotensina.

com SF apresentou maior incidência de elevação dos marcadores de lesão miocárdica periprocedimento comparativamente àqueles tratados com SNF (8,6\% vs. $4,0 \% ; \mathrm{P}<0,01)$

A taxa de ECAM, num acompanhamento clínico de $12,6 \pm 3,4$ meses, foi maior no grupo que recebeu SNF (9,5\% vs. 14,8\%; RR = 1,56; IC 95\% 1,07-2,27; P = 0,01). O óbito ao final do seguimento clínico também foi maior no grupo SNF $(2,8 \%$ vs. $6,7 \%$; RR $=2,41$; IC 95\% 1,22-4,77; P $<0,01)$, assim como o óbito de origem cardíaca $(1,7 \%$ vs. 3,7\%; RR = 2,78; IC $95 \% 1,16-6,68 ; P=0,01)$ e a RVA $(3,9 \%$ vs. $7,2 \%$; $R R=1,85 ;$ IC 95\% 1,03-3,35; $P=0,03)$. Por outro lado, não houve diferenças significativas entre o dois
TABELA 2

Características angiográficas e do procedimento

\begin{tabular}{|c|c|c|c|}
\hline Características & $\begin{array}{c}\text { SF } \\
(n=359)\end{array}$ & $\begin{array}{c}\text { SNF } \\
(n=580)\end{array}$ & $\begin{array}{l}\text { Valor } \\
\text { de } P\end{array}$ \\
\hline Vasos tratados, n (\%) & & & $<0,01$ \\
\hline Descendente anterior & $182(50,7)$ & $186(32,1)$ & \\
\hline Circunflexo & $64(17,8)$ & $103(17,7)$ & \\
\hline Coronário direito & $61(17)$ & $200(34,5)$ & \\
\hline Tronco de coronária esquerda & $1(0,3)$ & $9(1,6)$ & \\
\hline Enxerto de safena, n (\%) & $14(3,9)$ & $37(6,4)$ & 0,10 \\
\hline Bifurcação, n (\%) & $79(22)$ & $57(9,8)$ & $<0,01$ \\
\hline Oclusão total, n (\%) & $10(2,7)$ & $17(2,9)$ & 0,89 \\
\hline Reestenose, n (\%) & $61(17)$ & $15(2,6)$ & $<0,01$ \\
\hline $\begin{array}{l}\text { Número de vasos tratados } \\
\text { por paciente }\end{array}$ & $1,5 \pm 0,22$ & $1,0 \pm 0,50$ & $<0,01$ \\
\hline $\begin{array}{l}\text { Tratamento de } \\
2 \text { ou mais vasos, n (\%) }\end{array}$ & $91(25,3)$ & $33(5,7)$ & $<0,01$ \\
\hline Número stents por paciente & $1,48 \pm 0,5$ & $1,07 \pm 0,4$ & $<0,01$ \\
\hline Calibre dos stents, mm & $2,90 \pm 4,2$ & $3,10 \pm 4,3$ & 0,11 \\
\hline Extensão do stent, mm & $19,6 \pm 9,3$ & $20,5 \pm 6,6$ & 0,09 \\
\hline
\end{tabular}

grupos na incidência de IAM (1,7\% vs. 0,5\%; RR = 0,30; IC $95 \% 0,07-1,23 ; P=0,07)$ e AVE $(1,1 \%$ vs. $0,2 \%$; $R R=0,15 ;$ IC 95\% 0,01-1,37; P = 0,07) no mesmo período analisado (Tabela 3 ).

Os resultados das curvas de Kaplan Meier em relação à sobrevivência livre de ECAM, óbito, óbito de origem cardíaca e RVA estão discriminados na Figura, revelando diferenças significativas a favor dos SF.

Em relação à trombose dos stents definitiva ou provável, esta foi muito baixa em ambos o grupos (0 vs. $0,83 \%$; $P=0,06)$ no período de acompanhamento relativamente curto, previamente relatado.

A análise multivariada das variáveis pré-especificadas identificou apenas a doença renal crônica (IRC) $(R R=2,05 ;$ IC 95\% 1,40-2,98; $\mathrm{P}<0,01)$ e a SCA $(R R=2,08 ;$ IC 95\% 1,42-3,02; $\mathrm{P}<0,01)$ como preditores independentes para a ocorrência de ECAM na evolução tardia dos diabéticos (Tabela 4). O uso de SF não mostrou ser preditor de ECAM nesse grupo $(R R=1,35 ; I C$ 95\% 0,87-2,10; $P=0,17)$.

\section{DISCUSSÃO}

Em nosso estudo, que incluiu uma coorte de pacientes diabéticos não selecionados tratados por ICP em um hospital público terciário, observamos uma redução de ECAM, óbito, óbito de causa cardíaca e RVA no grupo tratado com SF. Entretanto, após o ajuste das variáveis de confusão, os SF não mostraram modificar 
TABELA 3

Eventos cardiovasculares ao final de 1 ano

\begin{tabular}{|c|c|c|c|c|c|}
\hline Evento & $\begin{array}{c}\text { SF } \\
(n=359)\end{array}$ & $\begin{array}{c}\text { SNF } \\
(n=580)\end{array}$ & $\mathbf{R R}$ & IC 95\% & Valor de $\mathrm{P}$ \\
\hline ECAM, n (\%) & $34(9,5)$ & $86(14,8)$ & 1,56 & $1,07-2,27$ & 0,01 \\
\hline Óbito, n (\%) & $10(2,8)$ & $39(6,7)$ & 2,41 & $1,22-4,77$ & $<0,01$ \\
\hline Óbito cardíaco, n (\%) & $6(1,7)$ & $27(3,7)$ & 2,78 & $1,16-6,68$ & 0,01 \\
\hline AVE, n (\%) & $4(1,1)$ & $1(0,2)$ & 0,15 & $0,01-1,37$ & 0,07 \\
\hline IAM, n (\%) & $6(1,7)$ & $3(0,5)$ & 0,3 & $0,07-1,23$ & 0,07 \\
\hline RVA, n (\%) & $14(3,9)$ & $42(7,2)$ & 1,85 & $1,03-3,35$ & 0,03 \\
\hline
\end{tabular}

SF: stents farmacológicos; SNF: stents não farmacológicos; RR: razão de risco; IC 95\%: intervalo de confiança de 95\%; ECAM: eventos cardiovasculares adversos maiores; AVE: acidente vascular encefálico; IAM: infarto agudo do miocárdio; RVA: revascularização do vaso alvo.

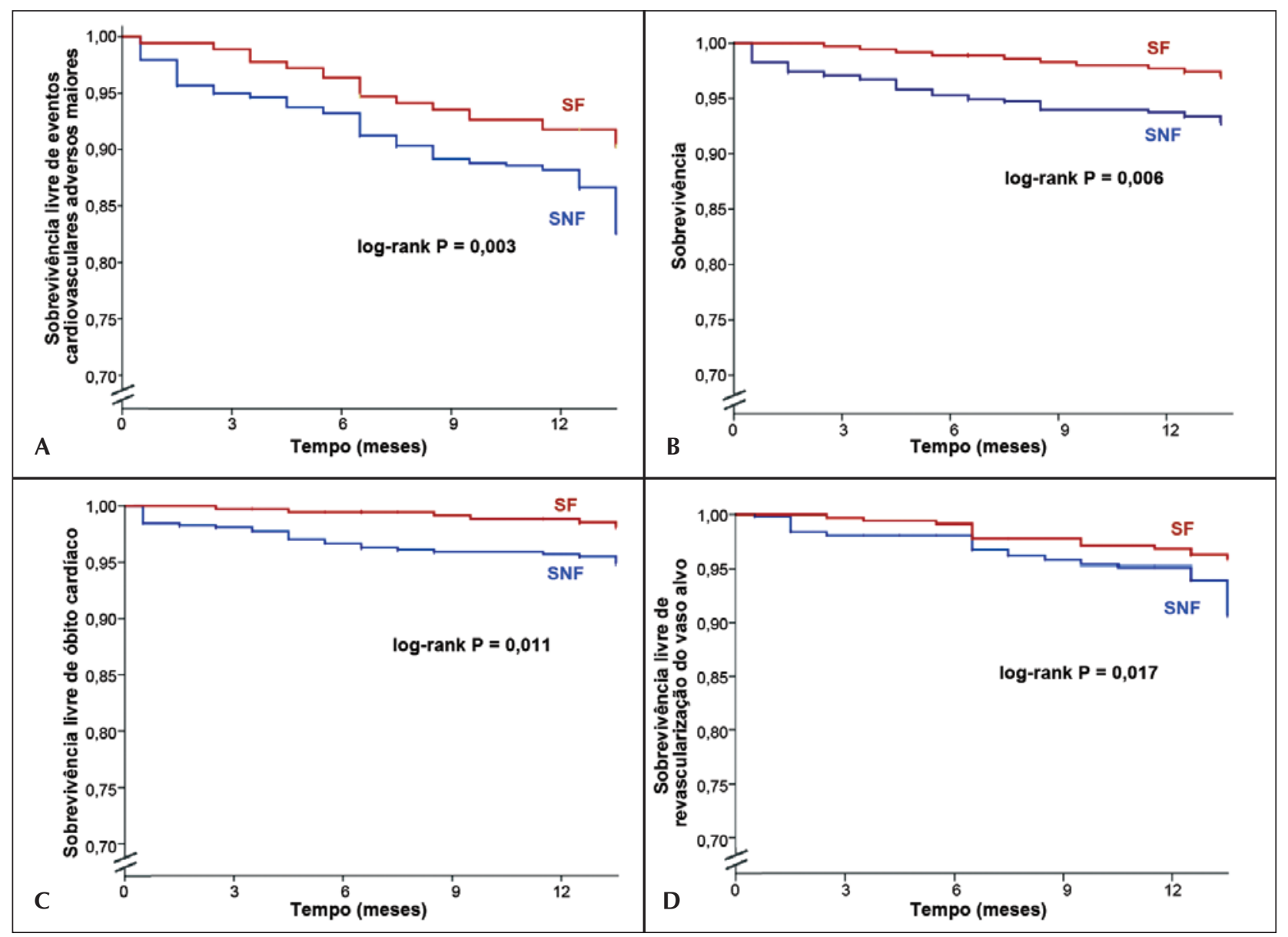

Figura. Curvas de sobrevivência livre de eventos cardiovasculares adversos maiores (A); curvas de sobrevivência (B); curvas de sobrevivência livre de óbito cardíaco (C); curvas de sobrevivência livre de revascularização do vaso alvo (D). SF = stent farmacológico; SNF = stent não farmacológico.

a ocorrência dos eventos adversos combinados. A doença renal crônica e a SCA foram as únicas variáveis pré-especificadas preditoras de pior evolução clínica.

Atualmente, o desenvolvimento tecnológico dos materiais disponíveis para a realização da ICP, aliado à vasta experiência dos operadores no manejo de pacientes de alto risco e à disponibilidade dos SF, permitiu a expansão das indicações do tratamento percutâneo, incluindo aqueles portadores de DAC de alta complexidade clínica e anatômica, como os diabéticos. ${ }^{16,17}$ 
TABELA 4

Preditores de eventos cardiovasculares adversos maiores em diabéticos

\begin{tabular}{lccc}
\hline & RR & IC 95\% & Valor de P \\
\hline Idade & 1,10 & $0,99-1,03$ & 0,08 \\
Sexo & 1,00 & $0,69-1,45$ & 0,97 \\
Uso de insulina & 0,96 & $0,60-1,54$ & 0,87 \\
Insuficiência renal crônica & 2,05 & $1,40-2,98$ & $<0,01$ \\
Síndrome coronária aguda & 2,08 & $1,42-3,02$ & $<0,01$ \\
ICP prévia & 0,80 & $0,48-1,33$ & 0,40 \\
Número de vasos tratados & 0,74 & $0,44-1,25$ & 0,27 \\
Insuficiência cardíaca & 0,61 & $0,32-1,13$ & 0,11 \\
Uso de SF & 1,35 & $0,87-2,10$ & 0,17 \\
IAM periprocedimento & 1,50 & $0,80-2,92$ & 0,19 \\
\hline
\end{tabular}

RR: razão de risco; IC 95\%: intervalo de confiança de 95\%; ICP: intervenção coronária percutânea; SF: stent farmacológico; IAM: infarto agudo do miocárdio.

Em nosso estudo, pudemos constatar que os diabéticos tratados com o emprego de SF corroboram esse cenário, na medida em que apresentaram características angiográficas mais desafiadoras, envolvendo frequentemente o tratamento da artéria descendente anterior (com maior área de miocárdio em risco de isquemia), lesões localizadas em bifurcações e reestenoses coronárias. Confirmando a complexidade das lesões tratadas, maior número de vasos foi abordado, resultando numa relação vaso tratado por paciente/número de stents por paciente significativamente maior no grupo de diabéticos revascularizados com SF. Tais características demandam a utilização de próteses mais eficientes, com eluição de fármacos que inibam, de forma efetiva, a proliferação neointimal, sendo possível reduzir a recorrência das obstruções devido à reestenose coronária e, consequentemente, a necessidade de novas revascularizações em pacientes já predispostos a esses eventos.

$\mathrm{Na}$ literatura, vários estudos comparando a utilização de SF vs. SNF em diabéticos e não diabéticos demonstram claramente a superioridade dos primeiros na redução significativa da reestenose coronária e, portanto, de nova abordagem de lesões recidivantes, reduzindo a exposição dos pacientes aos riscos inerentes de um novo procedimento, percutâneo ou cirúrgico.7,11,12

Também é interessante observar, em nossa população, a maior proporção de pacientes em uso de insulina no grupo do SF $(29,5 \%$ vs. $10,0 \%$; $P<0,01)$, denotando maior gravidade e duração do diabetes. Sabe-se que, em geral, esses pacientes podem apresentar doença aterosclerótica mais difusa, e intenso processo inflamatório das artérias coronárias e dos leitos vasculares em geral, o que resulta em maior predisposição a reestenose, trombose arterial e IAM. ${ }^{18-20}$ Publicação nacional recente ${ }^{21}$ investigou os resultados hospitalares de quase 2.000 diabéticos do registro Angiocardio, submetidos à ICP e divididos entre os tratados com insulina (21\%) e aqueles em uso de hipoglicemiantes orais. Os pesquisadores constataram que, apesar do maior porcentual de mulheres e de doença renal crônica no subgrupo em uso de insulina, os desfechos cardiovasculares maiores foram semelhantes nos dois grupos, sendo que o tratamento com insulina não foi preditor independente para a ocorrência de eventos adversos na fase hospitalar.

Registro realizado pelo National Heart, Lung and Blood Institute, em 2008, envolvendo mais de 2.500 pacientes diabéticos, com e sem uso de insulina, e tratados com SF e SNF, revelou que o uso de SF reduziu o risco de nova revascularização e também dos desfechos de segurança, como morte e IAM. Todavia, a redução da mortalidade e de IAM foi restrita aos diabéticos não tratados com insulina. ${ }^{21}$ Vale ressaltar que, em nossa pesquisa, a utilização de insulina não foi uma variável preditora de eventos cardiovasculares adversos no longo prazo.

No tocante aos resultados hospitalares, nossos achados mostraram taxas de sucesso do procedimento equivalentes entre os dois tipos de stents, assim como as de ocorrência de morte e AVE, confirmando a segurança e a eficácia dos SF na fase mais precoce pós-ICP. A maior incidência de elevação dos marcadores de lesão miocárdica peri-ICP provavelmente relaciona-se à maior complexidade anatômica das lesões tratadas no subgrupo dos SF, com maior de número de vasos abordados e stents implantados.

Quanto à trombose dos stents (definitiva ou provável, segundo critérios do ARC), esta foi muito baixa em ambos o grupos $(0,83 \%$ vs. 0$)$ num período de acompanhamento de 12,6 $\pm 3,4$ meses. Esses resultados são semelhantes aos do estudo ESSENCE-DIABETES II, realizado por Park et al. ${ }^{22}$, que demonstraram taxas de trombose subaguda e tardia em diabéticos submetidos ao implante de stents com eluição de zotarolimus e sirolimus $<1 \%$ ao final de 12 meses de evolução.

Em relação ao desfecho primário do estudo, constatamos que a ocorrência de ECAM foi significativamente menor naqueles diabéticos tratados com SF, o mesmo ocorrendo com o óbito de causa cardíaca e a RVA. À primeira vista, tais resultados parecem demonstrar que a utilização dos SF em diabéticos está relacionada à melhor evolução clínica em longo prazo, o que exige cautela na interpretação dos dados. Quando comparamos nossos resultado aos de outras pesquisas, verificamos que a maioria não demonstra redução significativa de mortalidade ou de outros desfechos cardiovasculares maiores nos pacientes tratados com SF, quer sejam eles diabéticos ou não. ${ }^{23,24}$

Alguns estudos revelam redução de trombose de stent e IAM, porém sem diminuir, de forma consistente, as taxas de mortalidade com emprego desse tipo de 
prótese, mesmo as categorizadas como de segunda ou terceira geração, assim chamadas devido à presença de novas plataformas metálicas, polímeros mais biocompatíveis e menos trombogênicos, e fármacos com alto poder de inibição neointimal. ${ }^{8,25}$

Outras pesquisas observacionais e registros internacionais, envolvendo grande número de pacientes diabéticos e não diabéticos tratados com SF, revelaram redução de desfechos duros, como morte e IAM, além, obviamente, das taxas de novas revascularizações. ${ }^{10,26,27}$ No entanto, tais resultados merecem uma reflexão e devem ser vistos com cautela em virtude de possíveis vieses de seleção dos pacientes e do tempo relativamente curto de acompanhamento clínico. Em nosso estudo, por exemplo, a análise multivariada dos preditores para a ocorrência de ECAM foram a doença renal crônica e a SCA, fato este fartamente comprovado na literatura. ${ }^{28}$ Coincidentemente, essas variáveis estavam significativamente mais presentes no grupo de pacientes tratados com SNF, o que desfavoreceu esse grupo e pôde explicar os melhores resultados obtidos nos pacientes submetidos ao implante de SF. Após ajuste da análise multivariada, observamos que não houve diferenças significativas nas taxas de ECAM, entre os pacientes tratados com e sem SF, ao final do seguimento clínico de 1 ano $(R R=1,35 ;$ IC 95\% 0,87-2,10; $P=0,17)$.

Pode-se, então, questionar, em nosso estudo, a opção pelo implante de SNF nos diabéticos portadores de SCA e insuficiência renal - comprovadamente pacientes de alto risco para ocorrência de eventos adversos. Algumas possíveis explicações são: (1) SF não estão rotineiramente disponíveis na maioria dos hospitais públicos ligados ao SUS; (2) a segurança e a eficácia da utilização de SNF para o tratamento de pacientes com quadros isquêmicos agudos, que requeiram ICP de urgência ou emergência, é equivalente às dos SF, pois, nesses casos, a prioridade é o tratamento da lesão culpada com o intuito de reduzir agudamente eventos como morte e IAM; (3) ainda em pacientes portadores de SCA, pode ser preferível o implante de SNF, devido a questões relacionadas ao não conhecimento detalhado da história clínica do paciente em termos de aderência à dupla antiagregação plaquetária ou mesmo à necessidade de suspensão precoce desta, em razão de comorbidades extracardíacas; (4) em pacientes renais crônicos, pode-se argumentar a favor do uso de SNF devido à eventual necessidade de outros procedimentos invasivos não cardiológicos, como implante de cateteres ou a realização de fístulas arteriovenosas para diálise e a possibilidade de transplante renal, situações nas quais a dupla antiagregação plaquetária pode predispor a sangramentos maiores.

\section{Limitações}

Trata-se de um estudo não randomizado, portanto sujeito a vieses de seleção na população pesquisada; o período de acompanhamento dos pacientes foi re- lativamente curto para a análise das reais diferenças na ocorrência de eventos cardiovasculares adversos em diabéticos; não obtivemos informações a respeito da aderência e do tempo de duração da dupla antiagregação plaquetária, fatores que poderiam interferir na incidência de eventos adversos, principalmente em pacientes tratados com SF e naqueles com SCA pré-ICP.

\section{CONCLUSÕES}

Em uma população constituída por pacientes diabéticos não selecionados, tratados por intervenção coronária percutânea, o uso de stents farmacológicos foi associado a taxas significativamente menores de desfechos cardiovasculares maiores, comparativamente aos stents não farmacológicos. Entretanto, após o ajuste das variáveis de confusão, o uso de stents farmacológicos não se mostrou preditor independente de eventos cardiovasculares adversos maiores no longo prazo.

\section{CONFLITO DE INTERESSES}

Os autores declaram não haver conflito de interesses relacionado a este manuscrito.

\section{AGRADECIMENTOS}

Gostaríamos de agradecer à equipe de estatísticos (João Italo França e Hayala Cristina de Souza), pela análise cuidadosa dos dados; à equipe do Centro de Pesquisa Clínica da Seção de Angioplastia Coronária (Renata Viana, Patrícia Paiva e Mayra Yagihara); e ao Sr. Reginaldo Barreto, pela disponibilização, organização e auxílio na coleta das informações do banco de dados do Serviço de Cardiologia Invasiva do Instituto Dante Pazzanese de Cardiologia.

\section{REFERÊNCIAS}

1. Luscher TF, Creager MA, Beckman JA, Cosentino F. Diabetes and vascular disease: pathophysiology, clinical consequences, and medical therapy: Part II. Circulation. 2003;108(13):1655-61.

2. Iijima R, Ndrepepa G, Mehilli J, Markwardt C, Bruskina O, Pache $J$, et al. Impact of diabetes mellitus on long-term outcomes in the drug-eluting stent era. Am Heart J. 2007;154(4):688-93.

3. Abizaid A, Kornowski R, Mintz GS, Hong MK, Abizaid AS, Mehran $R$, et al. The influence of diabetes mellitus on acute and late clinical outcomes following coronary stent implantation. J Am Coll Cardiol. 1998;32(3):584-9.

4. Jiménez-Navarro MF, Curiel E, Hernández-García JR, Alonso Briales JH, Domínguez Franco A, Gómez Doblas JJ, et al. Influence of diabetes mellitus on clinical outcome after percutaneous coronary revascularization. Rev Esp Cardiol. 2002; 55(4):365-71.

5. Lee TT, Feinberg L, Baim DS, Holmes DR, Aroesty JM, Carrozza JP Jr., et al. Effect of diabetes mellitus on five-year clinical outcomes after single-vessel coronary stenting (a pooled analysis of coronary stent clinical trials). Am J Cardiol. 2006;98(6):718-21.

6. Moses JW, Leon MB, Popma JJ, Fitzgerald PJ, Holmes DR, $\mathrm{O}^{\prime}$ Shaughnessy $\mathrm{C}$, et al. Sirolimus-eluting stents versus standard stents in patients with stenosis in a native coronary artery. $\mathrm{N}$ Engl J Med. 2003;349(14):1315-23. 
7. Sabate M, Jimenez-Quevedo P, Angiolillo DJ, Gomez-Hospital JA, Al-Fonso F, Hernandez-Antolin R, et al.; DIABETES Investigators. Randomized comparison of sirolimus-eluting stent versus standard stent for percutaneous coronary revascularization in diabetic patients: the diabetes and sirolimus-eluting stent (DIABETES) trial. Circulation. 2005; 112(14):2175-83.

8. Bangalore $\mathrm{S}$, Kumar S, Fusaro $\mathrm{M}$, Amoroso $\mathrm{N}$, Kirtane AJ, Byrne RA, et al. Outcomes with various drug eluting or bare metal stents in patients with diabetes mellitus: mixed treatment comparison analysis of 22,844 patient years of follow-up from randomised trials. BMJ. 2012;345:e5170.

9. de Waha A, Dibra A, Kufner S, Baumgart D, Sabate M, Maresta $\mathrm{A}$, et al. Long-term outcome after sirolimus-eluting stents versus bare metal stents in patients with diabetes mellitus: a patient-level meta-analysis of randomized trials. Clin Res Cardiol. 2011;100(7):561-70.

10. Minha S, Bental T, Assali A, Vaknin-Assa H, Lev El, Rechavia $\mathrm{E}$, et al. A comparative analysis of major clinical outcomes using drug-eluting stents versus bare metal stents in diabetic versus nondiabetic patients. Catheter Cardiovasc Interv. 2011;78(5):710-7.

11. Moussa I, Leon MB, Baim DS, O'Neill WW, Popma JJ, Buchbinder $M$, et al. Impact of sirolimus-eluting stents on outcome in diabetic patients: A SIRIUS (SIRolImUS-coated Bx velocity balloonexpandable stent in the treatment of patients with de novo coronary artery lesions) substudy. Circulation. 2004;109(19):2273-8.

12. Patti G, Nusca A, Di Sciascio G. Meta-analysis comparison (nine trials) of outcomes with drug-eluting stents versus bare metal stents in patients with diabetes mellitus. Am J Cardiol. 2008;102(10):1328-34.

13. Kappetein AP, Head SJ, Morice MC, Banning AP, Serruys PW, Mohr FW, et al.; SYNTAX Investigators. Treatment of complex coronary artery disease in patients with diabetes: 5 -year results comparing outcomes of bypass surgery and percutaneous coronary intervention in the SYNTAX trial. Eur J Cardiothorac Surg. 2013;43(5):1006-13.

14. Farkouh ME, Domanski M, Sleeper LA, Siami FS, Dangas G, Mack $M$, et al.; FREEDOM Trial Investigators. Strategies for multivessel revascularization in patients with diabetes. $\mathrm{N}$ Engl J Med. 2012;367(25):2375-84.

15. Cutlip DE, Windecker S, Mehran R, Boam A, Cohen DJ, van Es GA, et al.; Academic Research Consortium. Clinical end points in coronary stent trials: a case for standardized definitions. Circulation. 2007;115(17):2344-51.

16. Levine GN, Bates ER, Blankenship JC, Bailey SR, Bittl JA, Cercek B, et al. 2011 ACCF/AHA/SCAl Guideline for Percutaneous Coronary Intervention: a report of the American College of Cardiology Foundation/American Heart Association Task Force on Practice Guidelines and the Society for Cardiovascular Angiography and Interventions. Circulation. 2011;124(23): e574-651.
17. Wijns W, Kolh P, Danchin N, Di Mario C, Falk V, Folliguet $\mathrm{T}$, et al. Guidelines on myocardial revascularization. Eur Heart J. 2010;31(20):2501-55.

18. Iakovou I, Schmidt T, Bonizzoni E, Ge L, Sangiorgi GM, Stankovic G, et al. Incidence, predictors, and outcome of thrombosis after successful implantation of drug-eluting stents. JAMA. 2005; 293(17):2126-30.

19. van Werkum JW, Heestermans AA, Zomer AC, Kelder JC, Suttorp MJ, Rensing BJ, et al. Predictors of coronary stent thrombosis: the Dutch Stent Thrombosis Registry. J Am Coll Cardiol. 2009;53(16):1399-409.

20. Centemero MP, Stadler JR. Stent thrombosis: an overview. Expert Rev Cardiovasc Ther. 2012;10(5):599-615.

21. Farinazzo MM, Cantarelli MJ, Castello Jr HJ, Gonçalves R, Giopatto S, Guimarães JBF. Intervenção coronária percutânea em diabéticos tratados com insulina. Rev Bras Cardiol Invasiva. 2013;21(1):43-8.

22. Park GM, Lee SW, Park SW, Kim YH, Yun SC, Cho YR, et al. Comparison of zotarolimus-eluting stent versus sirolimuseluting stent for de novo coronary artery disease in patients with diabetes mellitus from the ESSENCE-DIABETES II trial. Am J Cardiol. 2013;112(10):1565-70.

23. Simsek C, Räber L, Magro M, Boersma E, Onuma $Y$, Stefanini $G G$, et al. Long-term outcome of the unrestricted use of everolimus-eluting stents compared to sirolimus-eluting stents and paclitaxel-eluting stents in diabetic patients: the Bern-Rotterdam diabetes cohort study. Int J Cardiol. 2013;170(1):36-42.

24. Stenestrand U, James SK, Lindback J, Frobert O, Carlsson J, Schersten F, et al. Safety and efficacy of drugeluting vs. bare metal stents in patients with diabetes mellitus: long-term follow-up in the Swedish Coronary Angiography and Angioplasty Registry (SCAAR). Eur Heart J. 2010;31(2):177-86.

25. Garg P, Normand SL, Silbaugh TS, Wolf RE, Zelevinsky K, Lovett A, et al. Drug-eluting or bare-metal stenting in patients with diabetes mellitus: results from the Massachusetts Data Analysis Center Registry. Circulation. 2008;118(22):2277-85, $7 p$ following 2285 .

26. Mulukutla SR, Vlachos HA, Marroquin OC, Selzer F, Holper EM, Abbott JD, et al. Impact of drug-eluting stents among insulin-treated diabetic patients: a report from the National Heart, Lung, and Blood Institute Dynamic Registry. JACC Cardiovasc Interv. 20081(2):139-47.

27. Kirtane A, Gupta A, lyengar S, Moses J, Leon M, Applegate $R$, et al. Safety and efficacy of drug-eluting and bare metal stents: comprehensive meta-analysis of randomized trials and observational studies. Circulation. 2009;119(25):3198-206.

28. Lehmann R, Fichtlscherer S, Schächinger V, Held L, Hobler C, Baier G, et al. Favorable long-term survival in patients undergoing multivessel- $\mathrm{PCl}$ compared to predicted prognosis of CABG estimated by EuroSCORE: procedural and clinical determinants of long-term outcome. J Interv Cardiol. 2009; 22(6):511-9. 